\title{
FILTRAGE RYTHMIQUE DES DONNÉES ACOUSTIQUES EN PAROLE SPONTANEE: UN TRAITEMENT POUR LA FO ET LA DURÉE SEGMENTALE
}

\author{
I. GUAÏTELLA \\ Institut de Phonétique, Université de Provence, 29 Av R. Schuman, F-13621 Aix-en-Provence, France
}

\begin{abstract}
In spontaneous speech, the study of intonation postulate to call into question the stereotypes stemming from the studies of reading materials. From our point of view, the place of accent is not predictable, but it can be detected according to rhythmic cues. Fundamental frequency seems to be the essential one which a duration cue can be optionaly added. The presence/absence of duration cues enables us to build thythmic hierarchy based upon perception processes. Duration lenghtening alone, i.e. without any to cue, often corresponds to a phenomenon called "vocal hesitation". In this paper, we try to show both acoustic behavior and rhythmic and perceptual functions of prosody in spontaneous speech.
\end{abstract}

\section{Introduction}

Dans l'étude de l'intonation (comme peutêtre dans toute étude de faits de communication) nous pouvons appréhender les phénomènes selon deux points de vue. Ceux-ci correspondent globalement aux axes paradigmatique et syntagmatique et posent, pour le premier, le problème de la description en termes de signes, et, pour le second, le probleme de la description en termes de segmentation d'un continuum temporel (Bolinger 1985). Une courbe intonative interrogative, par exemple, peut etre décrite comme un signe, ou symbole, ayant une autonomie ef faisant l'objet d'un choix. Ainsi, cette courbe pourra commuter avec une courbe affirmative. La même courbe pourra également être considérée en fonction de son apport à la structuration rythmique du continuum de parole au sein duquel elle s'insère afin de le rendre perceptible (Darwin 1975; "Guide function" de Barry 1981). Dans cette perspective, la signification symbolique de la courbe est secondaire, ce qui devient important est le fait que cette coube soit située temporellement à un moment donné, et intervienne ainsi dans l'organisation rythmique. Nous avons, pour des raisons de méthode, séparé les perspectives rythmique et symbolique dans notre étude de l'intonation. Nous nous contenterons ici (pour des raisons de place), de définir comment la courbe mélodique, de par ses caractéristiques dynamiques, contribue à organiser rythmiquement le discours.

Quelles relations la fréquence fondamentale entretient-elle avec l'organisation rythmique de la parole? De nombreux auteurs considèrent que la fréquence fondamentale est la principale composante orale du rythme de la parole, cependant appréhender le continuum des variations de la mélodie comme une composante rythmique, suppose de se poser plusieurs questions. Notre point de vue étant que la localisation de l'accent n'est pas prédictible par des règles issues de composantes verbales (Bolinger 1972), le problème est de savoir comment situer les syllabes mises en relief. La détermination de l'organisation rythmique à partir des indices devient alors un passage obligatoire sans lequel nous ne saurions parter d'analyse rythmique.

\section{Specification des problèmes et des hupotheses}

1-1-Comment décrire l'organisation ryttmique d'un mouvement continus?

Le rythme suppose une série d'éléments discrets entretenant des relations réciproques. II va donc être nécessaire de retrouver cette série d'éléments discrets à l'intérieur d'un mouvement continu (Malmberg 1967). Si une courbe se réalise sur plusieurs syllabes, nous pouvons considérer que ces syllabes constituent la suite d'éléments discrets (cependant nous pourrions également découper le continuum temporel en segments de durée égale).

\section{1-2-Apport de la theorie de l'information}

La théorie de linformation propose des outils conceptuels pour répondre à ce type de question. Si nous considérons une courbe, sans même chercher à la segmenter en unités de taille inférieure (syllabes ou segments temporels), nous pouvons voir, grâce à l'analyse en termes d"information", que cette courbe dispose d'une dynamique rythmique interne. Imaginons un continuum de fréquence fondamentale. Si nous en isolons un fragment, nous pouvons constater que l'apport d'information est constitué par le démarrage, l'arrêt ou les changements de sens de cette courbe et par le niveau fréquentiel ou ceux-ci se réalisent. Nous considérons que ces changements dans la trajectoire participent en tant qu'indices à l'organisation rythmique. Leurs positions par rapport à la chaîne segmentale permettra de déterminer les syliabes mises en relief. Cette méthodologie, partant du principe de détermination de l'accent à partir des indices, est en concordance avec les modéles de Bolinger (1972, 1985), de Garding (1977) et de Kohler (1991). 


\section{1-3- Apport dinformation pour le rythme ou pour le sens?}

II est évident que la capacité potentielle de la courbe à générer des accents par focalisation (nous définissons le terme "focalisation" par: participation d'indices à la mise en relief d'un élément segmentan), est liée à la nécessité de mettre en valeur, pour des raisons d'ordre pragmatique evou sémantique, certains éléments de la chaîne verbale. Cependant, it ne s'agit pas pour nous de justifier la localisation d'un quelconque accent par des arguments liés à la volonté du locuteur, mais de déterminer simplement cette localisation. Nous pensons que la motivation de la localisation de l'accent dépend de l'ensemble des composantes pragmatiques qui préside à la génération de la parole. En ce sens, expliquer la présence d'un accent revient à expliquer la totalité des conditions pragmatiques qui déterminent un acte de parole. Nous ne contestons pas que la focalisation d'une syllabe soit liée au sens (du message), mais nous considérons qu'elle est également liée à la totalité des constituants pragmatiques, et, de ce fait, il est extrêmement complexe d'expliquer réellement et objectivement, pourquoi l'une ou l'autre syllabe est focalisée. Ceci supposerait, en effet, une connaissance objective et totale de toute la situation, ce que le locuteur lui-même ne détient pas de façon consciente.

\section{1-4- Probabilités et contraintes}

Par rapport au modèle d'interprétation proposé par la théorie de linformation, notre méthodologie présente des lacunes qu'il sera nécessaire de combler. Parmi celles-ci, la notion de probabilité d'apparition d'un phénomène. Or, cette notion est très délicate à manipuler en prosodie, il s'agit en effet de déterminer ce qui est prévisible et ce qui ne l'est pas dans l'évolution de la fréquence fondamentale. Etant donné que la fréquence fondamentale est directement contrainte par la production physique de la voix, on peut supposer que tout ce qui, dans la fréquence fondamentale, est conditionné physiologiquement, apparaît comme prévisible. Par exemple, le lait que l'intonation ne puisse pas monter indéfiniment est conditionné physiologiquement, et est, par conséquent, prévisible pour le locuteur/auditeur possédant une connaissance empirique de ce phénomène. Autre exemple: pour produire deux montées successives, il est bien entendu nécessaire de produire une descente (ou un silence), ceci est également prédictible puisque le locuteur en a une connaissance a priori. Or, pour l'ensemble de ces phénomènes, il est difficile d'évaluer la limite de ce qui est contraint, et donc prédictible, et de ce qui fait l'objet d'un choix expressif, et est donc réellement porteur d'information. Ce problème nous semble pourtant essentiel, puisqu'il nous amène à appréhender les phénomènes intonatifs en tant que trace d'un "conflit" entre volonté expressive el contrainte physiologique, la première étant non-prédictible, la seconde prédictible, ce conflit s'incarnant dans l'évolution du système, perturbé par les conditions externes mais inéluctables, qui régissent son fonctionnement. Ainsi, les facleurs externes (situation de parole, émotion...) agissent sur le système prosodique. Sans ces facleurs, la parole perdrait son caractère "informatif".

\section{1-5- Le traitement de la durée syllabique}

La modification de durée syllabique est altestée par de nombreuses études comme étant un phénomène essentiel de la manifestation du rythme. Or, notre travail sur la durée de syllabes accentuées en lecture de phrases (Guailella 1988, 1991), nous amène à considérer que les modifications de durée des syllabes accentuées ne consistent pas seulement en un allongement mais parfois en un raccourcissement. Comment, donc, déterminer la présence dun accent à partir des indices fournis par la durée syllabique, si celle-ci peut varier à la fois dans le sens de l'allongement et du raccourcissement?

Nous avons tenté de répondre à cette question à partir des deux principes suivants:

- la pause silencieuse apparaît comme une remise à zéro de la valeur syllabique de rétérence. Après chaque pause silencieuse il sera donc nécessaire de reconsidérer le rapport entre durée syllabique de référence (durées des syllabes atones) et durées particulières (durées des syllabes accentuées). Ce principe nous permet de faire face aux problèmes de variations globales de la durée syllabique, engendrant des modifications dans le pourcentage de variation de durée entre syllabes alones et syllabes accentuées. Cette procédure se justifie par le lait que la pause silencieuse, par sa durée propre, permet l'effacement dans la mémoire à court terme de la trace de la durée syllabique de référence correspondant à ce qui précède la pause. En outre, nous pensons que la pause est nécessaire pour que le loculeur lui-même parvienne à établir une nouvelle valeur de rélérence.

- Suite à une pause silencieuse, nous considérons donc le pourcentage de variations entre la première et la deuxième syllabe. Si ce pourcentage est intérieur à $20 \%$ (Rossi 1972, Klatt 1976), nous considérons alors la variation entre la deuxième et la troisième syllabe, et ainsi de suile jusqu'à ce que nous disposions d'une variation supérieure à $20 \%$. Dans ce cas, nous considérons qu'il s'agit de l'établissement du "pourcentage de variation référentielle", et nous appliquons ensuile ce pourcentage pour chaque syllabe par rapport à la syllabe qui précéde, jusqu’à la pause silencieuse suivante, afin de déterminer si la durée syllabique est un indice accentuel ou non.

Exemple: segmentation syllabique avec durées des syllabes et indices de durée (représenté par [+])

\# [c'est-à] - $219 \mathrm{~ms}$

[dire] - $212 \mathrm{~ms}$ (la différence de durée avec la syllabe précédente est intérieure à 20\%)

[que] - $140 \mathrm{~ms}$ (la différence de durée entre cette syllabe (s2) et la syllabe précédente (s1) est supérieure à 20\%. Celte différence va permettre d'établir la valeur de référence pour comparer les durées syllabiques, la valeur de référence sera égale dans ce cas à $s 1 / \mathrm{s} 2=1.5)[+]$

[les] -114

[mal] - 204

[gaches] -229

[sont] -168

[cons] -180

[tem] -147

[ment] - $211[\mathrm{t}]$

[tour] $-140 \quad[+]$

[nés] - 123

[vers] -127

[la] - 140

[france] - $495[+]$ 
L'ensemble de cette procédure - la référence à la pause qui précéde et aux durées syllabiques initiales - est régie par le principe de sélection à gauche des composantes de rérérence. Ceci est donc applicable, selon nous, à la parole spontanée (Guaîtella 1991).

\section{2-Etude dun cormus: analuse ruthmique systematique}

\section{2-1- La combinaison des Indices}

Les indices fournis par la fréquence fondamentale cévénement dans la courbe de to, localisé à l'intérieur d'un segment syllabique) el par la durée syllabique doivent être gérés afin de permettre la détermination de l'organisation rythmique de la parole. Selon nous, l'indice premier, sans lequel il ne peut y avoir d'accent, est la fréquence fondamentale. Nous suivons en cela la perspective déjà longuement développée par de nombreux phonéticiens. Nous considérons que la syllabe a plus de chance d'êire perçue comme accentuée par l'auditeur lorsque l'indice de fréquence fondamentale est accompagnée d'un indice de durée. Lorsqu'il y a seulement indice de durée mais pas d'indice de fréquence fondamentale, la syllabe n'est pas perçue comme accentuée, c'est ce qui explique que les hésitations - dont les durées correspondent apparemment à un indice accentuel, mais qui sont déconnectées du verbal - ne soient pas perçues comme accentuées (voir plus bas). Lorsque lindice foumi par la fréquence fondamentale n'est pas accompagné d'un indice de durée, nous considérons que la syllabe se trouve abrs à un niveau inférieur dans la hiérarchie perceptive des indices accentuels.

\section{2-2- L'organisation rythmique du corpus déterminée à partir des indices}

La transcription ci-dessous (extrait du corpus "Madagascar" consitué par une interview, voir Guaîtella 1991) permet de juger quelles syllabes ont été déterminées comme accentuées à partir de la méthode de détection des indices. Cette méthode a permis d'établir deux niveaux dans la potentialité qu'a une syllabe d'être perçue comme accentuée. Le premier niveau (représenté par la présence de + sous la syilabe) correspond aux syllabes déterminées par un indice fourni par la fréquence fondamentale et par un indice de durée. Le deuxième niveau (représenté par $(+))$ correspond aux syllabes déterminées uniquement par un indice de fréquence fondamentale, celles-ci sont donc inférieures dans la hiérarchie (perceptive) dans le sens où elles sont plus susceptibles de ne pas être perçues. Notre transcription est orthographique (et non pas phonétique) afin d'en faciliter la lecture. Lorsque plusieurs syllabes graphiques sont réalisées oralement comme une seule syllabe, nous notons ce phénomène de la façon suivante (exemple: [qui ont] notation utilisée pour la chaine phonétique [kjo]]).

je pense que c'est pas une empreinte c'est un manque d'empreinte (rires) \#

(t) $(+)$

c'est-à-dire que \#

$+(+)$

la france a exporté toutes les ressources du pays \#
$(+) \quad+(+)(+)(+)(+)$
$+(t)+$
$(+)(+)(+)$

sans apporter en échange \#

$(+)$

$+\quad+$

aucune infrastructure économique \#

$+(+)(+)+(+)(+)+$

c'est-à-dire pas de route pas de chemin [de fer] pas d'usine \#

$(+)$

$(+)+++$

[ou a]lors [ce qu'il]y avait a été détruit au départ des français \#
$(+)+$
(t)
$(+)(+)$
$(+)+$
$+$

contrairement à d'autres pays où il y a quand même eu des choses [qui ont] été
$+(+)$
$(+)$
$+(+)+$
$+$
$+++$
$(+) \quad(+)$

construites pour le pays lui-même \#

$(+) \quad(+)$

Pour les cas où plusieurs syllabes accentuées se suivent, nous proposons les interprélations suivantes:

- il peut s'agir dun phénomène de surcharge lié à des motivations expressives diverses

ex: "in industrialiser le pays"

/ in / in / dus / tria / li / ser / le / pay / ys /

$+++++$

Ces cas correspondent à ce que nous avons l'habitude de transcrire de la façon suivante: "EX-TRA-OR-DI-NAI-RE", c'eslà-dire une volonté de renforcer son propos par une mise en relief de toutes les syllabes. Bien entendu cette mise en relief de tous les segments se détériore elle-même de par la banalisation de l'accentuation qui produit une déperdition d'information.

- lorsque deux syllabes accentuées se situent l'une à la fin d'un mot et l'autre au début du mot suivant, on assiste apparemment à la création d'une frontière. Les principes rythmiques observés par Fraisse (1974) établissent que les accents sont 
positionnés aux bornes des groupements. Dans notre cas, la présence de deux accents à la suite tend à faire percevoir le premier comme la fin diun groupement et le deuxième comme le debut d'un autre groupement. Cette interprétation nous semble altestée par la présence de ce phénomène lors d'érumérations, où il confirme la segmentation en ternes énumératifs.

ex: "ils meurent de faim il y a les maladies"

/ ils / meurent / de / faim / ilya / les / ma / la / dies /

La présence d'un indice de durée (dans le sens de l'allongement) sans la présence d'un indice fourni par la fréquence fondamentale coìncide avec la réalisation des hésitations vocales. C'est-à-dire que toutes les syllabes comportant un indice d'allongement ne sont pas perçues comme des hésitations, mais que les hésitations correspondent à cette configuration, en précisant qu'elles font l'objet d'un accroissement extrême de la durée. Nous prétendons rendre compte des "potentialités rythmiques" contenues dans ce fragment de discours. Cependant, ces potentialités seront probablement l'objet d'un filtrage perceptif different suivant les auditeurs. Ainsi, nous pouvons établir des grilles rythmiques en fonction de ce qui est produit par le locuteur, mais on pourrait aussi, dans un deuxième temps, étabiir des grilies perceptives qui rendent compte du "comportement perceptif" des divers auditeurs. Ces grilles se fonderaient sur des faits d'alternance de perception d'une figure sur un fond.

\section{3-Place des hesitations vocales dans le systeme}

Si l'on utilise la méthodologie, décrite ci-dessus, de détermination de la place de l'accent à partir des indices, on s'aperçoit que l'hésitation se caractérise par un indice significatif et spécifique d'allongement de la "syllabe", en l'absence d'indice fourni par la fréquence fondamentale. L'allongement de la durée syllabique sans changement dans la fréquence fondamentale, ne serait donc pas un indice d'accentuation, mais - du moins pour les cas d'accroissement extrẻme - un indice d'une autre catégorie segmentale: l'hésitation. Nous pensons qu'il existe (au moins) trois unités au niveau segmental - pause, hésitation, syllabe -, et que ceci est confirmé par lutilisation du paramètre de durée qui permet la différenciation de ces trois catégories du point de vue perceptif (Guaîtella 1991, 1992).

Le paramètre de durée apparaît comme un indice accentuel, mais demeure uniquement un indice de second plan associé à la présence d'une modification de fo. L'existence du phénomène d'hésitation confirme le rôle primordial du paramètre de to pour la perception de l'accent. La description des potentialités rythmiques en fonction des indices produits contime que la perception assume une fonction active dans la détermination de l'organisation rythmique, et que l'on peut concevoir la coexistence de perceptions rythmiques différentes d'un mème énoncé.

\section{Concision}

Nous considérons donc que la prosodie, en parole spontanée, peut se décrire, de façon primaire, comme un système indépendant disposant de quatre composantes, lesquelles utilisent ou non le changement de sens de la courbe intonative, la durée et la montée ou la chute intonative. Nous considérons qu'il n'y a pas d'unités privilégiées pour l'interprétation de l'intonation en parole spontanée. Certaines unités peuvent être le cadre d'une analyse intonative (par exemple les réitérations, les tours de parole, les groupes de souffle, etc, voir Guaïtella 1991). Mais il s'agit là d'unités définies à d'autres niveaux et à l'intérieur desquelles on peut décrire ce qui se passe du point de vue intonatif. Nous pensons qu'il n'existe pas de constituant intonatif que l'on puisse considérer comme autonome (en dehors peut-être des clichés mélodiques). Par contre, les contours diposent d'un potentiel rythmique et symbolique (signes élémentaires constitués par les variations paramétriques: changement de pente...). Le caractère imprédictible de la prosodie en parole spontanée a, selon nous, deux conséquences: on ne saurait prévoir avec justesse ce qu'un locuteur va produire, par contre il est possible de dégager des règles globales de fonctionnement qui permettent de simuler de la parole spontanée en synthèse de la parole (Guaitella, Santi 1992).

\section{REFERENCES}

Barry W.J., 1981, "Prosodic Functions Revisited Again!", Phonetica, 38, 5-6, 320-40.

Bolinger D., 1972, "Accent is predictable (if you're a mind-reader)", Language, 48, 633-44.

Bolinger D., 1985, intonation and its parts, Edward Amold.

Darwin C.J., 1975, "On the dynamic use of prosody in speech perception", in: Structure and Process in Speech Perception, Cohen and Nooteboom (eds), Springer-Verlag, 178-94.

Fraisse P., 1974, Psychologie du nythme, P.U.F.

Garding E., 1977, "The importance of turning points for the pilch patterns of swedish accents", Scopil, 4, 27-36.

Guaïtella I., 1988, "Variations de durée en syllabe accentuée", Travaux de l'Instifut de Phonétique d'Aix., 12, 185-204.

Guaitella I., 1991, Ryhtme et parole: comparaison critique du rythme de la lecture oralisée et de la parole spontanée, Thèse de Doctorat, aix en-Provence.

Guaiitella I., 1992 (sous presse), "Hésitations vocales en parole spontanée: réalisations acoustiques et fonctions rythmiques", Travaux de l'I.nstitut de Phonétique d'Aix., 14.

Guaittella I., Sant S., 1992 (sous presse), "The punctuation and perception of read and spontaneous prosody: an application to speech synthesis", in: Talking Machines, Bailly \& Benoit (eds), Elsevier North-Holland.

Klatt D.H., 1976, "Linguistic uses of segmental duration in English: acoustic and perceptual evidence", J. Acoust. Soc. Am., 59(5), 1208-21.

Kohler K.J., 1991, "Prosody in speech synthesis: the interplay between basic research and TS application", Joumal of Phonetics, 19 , 1, 121-38.

Malmberg B., 1967, "Le rythme comme phénomène linguistique et phonétique", Actes du Congrès Les Rythmes, Lyon, 35-45.

Rossl M., 1972, "La perception de la durée et ses implications phonétiques", Travaux de l'lnstitut de Phonétique d'Aix., 1, 151 -64. 